\title{
Upregulation of MANCR predicts poor survival in patients with gastric cancer
}

\author{
LING YAO $^{1}$, JINHUA YAN $^{2}$, LIHONG GAN $^{1}$, SHENAN HUANG $^{1}$, FEI CHENG $^{1}$ and NIAN FANG ${ }^{1}$ \\ Departments of ${ }^{1}$ Gastroenterology and ${ }^{2}$ Hematology, The Third Affiliated Hospital of Nanchang University, \\ Nanchang, Jiangxi 330008, P.R. China
}

Received February 16, 2019; Accepted September 24, 2019

DOI: $10.3892 / \mathrm{ol} .2019 .11026$

\begin{abstract}
A previous study revealed that MANCR (mitotically associated long non-coding RNA) is implicated in breast cancer. The present study investigated the potential role of MANCR in gastric cancer (GC) and revealed that MANCR was upregulated in GC tissues compared with non-cancerous tissues. MANCR expression was not affected by clinical stages and a high MANCR expression level was associated with poor survival time. MicroRNA (miR)-101 was downregulated in cancer tissues compared with non-cancerous tissues and was inversely associated with MANCR expression. MANCR overexpression in GC cell lines in vitro resulted in miR-101 downregulation; however, miR-101 overexpression did not alter MANCR expression. Furthermore, MANCR overexpression promoted, while miR-101 overexpression inhibited GC cell proliferation. In addition, miR-101 overexpression decreased the effect of MANCR overexpression. The results obtained in the present study revealed that MANCR expression was associated with the survival of patients with GC, and MANCR overexpression in vitro may promote GC by downregulating miR-101 and increasing the proliferation of GC cells.
\end{abstract}

\section{Introduction}

With advances in cancer treatment and prevention, the survival of patients with gastric cancer (GC) has significantly improved during the past decades (1). However, GC remains a common type of malignancy worldwide and is a major cause of mortality among patients due to its aggressive nature (2). In effect, GC has been the leading cause of cancer-associated mortalities in China for the past 3 decades (3). A major challenge in the treatment of GC is the high prevalence of metastasis by the time of initial diagnosis (4). It has been reported that a

Correspondence to: Dr Nian Fang, Department of Gastroenterology, The Third Affiliated Hospital of Nanchang University, 128 Xiangshan North Road, Nanchang, Jiangxi 330008, P.R. China E-mail: qz56671@163.com

Key words: gastric cancer, mitotically associated long non-coding RNA, microRNA-101 considerable number of tumor suppressors and oncogenes that are involved in the pathogenesis of GC, and these genes may serve as potential therapeutic targets for GC (5). However, the tumor suppressors and oncogenes identified so far may not be sufficient to explain the complex mechanism underlying the pathogenesis of GC.

Long non-coding RNAs (lncRNAs) are transcripts $>200$ nucleotides in length that have been identified to serve important roles in cancer biology $(6,7)$. IncRNAs participate in the development and progression of cancer through the regulation of gene expression at different levels $(8,9)$. Therefore, it is possible to target lncRNAs to indirectly regulate the expression of specific genes involved in human diseases, thereby assisting disease treatment (10). However, the function of the majority of lncRNAs in cancer remains unknown. Therefore, future studies investigating the roles of lncRNAs in different aspects of cancer biology are required. A previous study reported that mitotically associated long non coding RNA (MANCR) affects cell division and genomic stability in breast cancer (11). In breast cancer, MANCR is upregulated and expression levels of MANCR are closely associated with cell cycle progression (11). LncRNA may interact with different downstream protein-coding genes or other non-coding RNAs, including microRNAs (miRNAs/miRs) (10). miR-101 is downregulated in GC and inhibits cancer cell invasion and migration in vitro (12). miR-101 and MANCR were inversely associated in GC (revealed by deep sequencing data, data not shown). The present study was therefore carried out to investigate the interaction between miR-101 and MANCR in GC.

\section{Materials and methods}

Patients. A total of 102 pateints were diagnosed and treated for GC in The Third Affiliated Hospital of Nanchang University between July 2011 and July 2013. The present study selected 69 cases (39 males and 30 females; age range, 35-67 years; mean age, $49.9 \pm 7.1$ years) based on the following inclusion criteria: i) Patients who had not received any therapy prior to admission, ii) patients who were diagnosed for the first time, iii) patients with no previous history of maligancy and iv) patients willing to participate in a five-year follow-up. The exclusion criteira were as follows: i) Patients with other medical disorders and ii) patients who were transferred from other hospitals. Accoring to the American Joint Committee on Cancer staging 
A

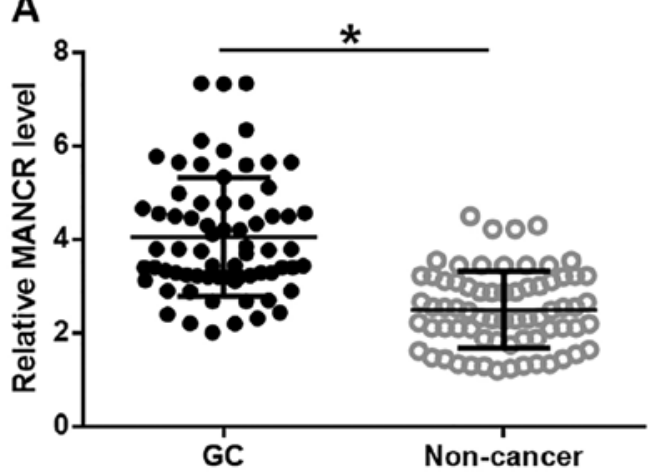

B

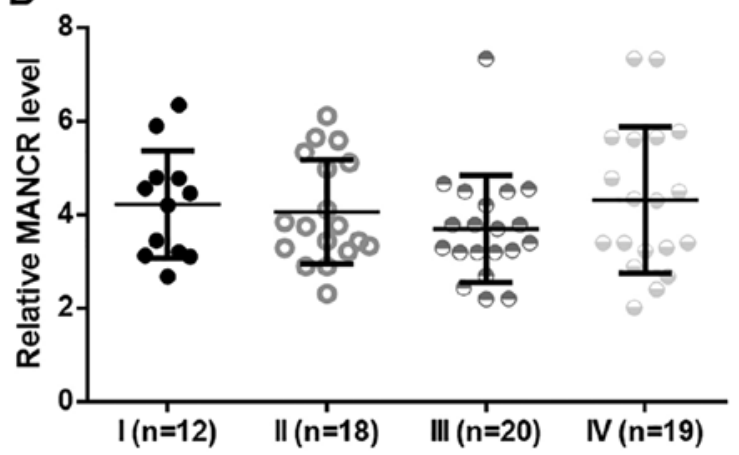

Figure 1. MANCR was upregulated in patients with GC but not affected by clinical stages. (A) Analysis of MANCR expression data using the paired t-test revealed that MANCR was significantly upregulated in GC tissues compared with adjacent non-cancerous tissues. (B) The one-way ANOVA and Tukey post hoc test revealed no significant differences in the expression level of MANCR in GC tissues in patients with different clinical stages. "P<0.05. MANCR, mitotically associated long non-coding RNA; GC, gastric cancer.

criteria (13), there were $12,18,20$ and 19 patients with stage I, II, III and IV GC, respectively. The Ethics Committee of The Third Affiliated Hospital of Nanchang University approved the study prior to patient enrolment and written informed consent was obtained from all patients.

Tissue specimens and cell lines. Patients underwent a gastric biopsy to collect $\mathrm{GC}$ and adjacent $(2 \mathrm{~cm})$ non-cancerous tissues prior to receiving therapy. All tissue specimens were analyzed by five experienced histopathologists. Tissue specimens were stored in liquid nitrogen prior to use.

The human GC cell lines HGC-27 (Sigma-Aldirch; Merck KGaA), and SNU-1 (American Type Culture Collection) were used for in vitro experiments. Cells were cultured in Eagle's Minimum Essential Medium (EMEM) containing $2 \mathrm{mM}$ glutamine, $1 \%$ non-essential amino acids and $10 \%$ fetal bovine serum supplemented with $100 \mathrm{IU} / \mathrm{ml}$ penicillin-streptomycin. All medium and cell culture reagents were from Sigma-Aldrich (Merck KGaA). Cell culture conditions were at $37^{\circ} \mathrm{C}, 5 \% \mathrm{CO}_{2}$ and $95 \%$ humidity, and were collected once they reached $\sim 80 \%$ confluency.

Follow-up. All patients were monitored for five years. Follow-up was performed through either outpatient visits or telephone calls. Patients who were lost to follow-up or succumbed to accidents or unrelated clinical disorders were not included in the present study.

Reverse-transcription quantitative polymerase chain reaction $(R T-q P C R)$. TRIzol ${ }^{\circledR}$ reagent (Invitrogen; Thermo Fisher Scientific, Inc.) was used to extract total RNA from GC and adjacent non-cancerous tissues and HGC-27 and SNU-1 cell lines. For MANCR RT-qPCR, AMV Reverse Transcriptase (Promega Corporation) was used to perform reverse transcription through following conditions: $50^{\circ} \mathrm{C}$ for $20 \mathrm{~min}$ and $80^{\circ} \mathrm{C}$ for $10 \mathrm{~min}$. All operations were performed according to the manufacturer's protocols. qPCR was subsequently performed using SYBR ${ }^{\circledR}$ Green Master Mix (Thermo Fisher Scientific, Inc.), according to the manufacturer's protocols. 18S rRNA was used as an endogenous control. miRNA extraction was performed using the mirPremier ${ }^{\mathrm{TM}}$ microRNA Isolation kit (Sigma-Aldrich; Merck KGaA). TaqMan MicroRNA Reverse

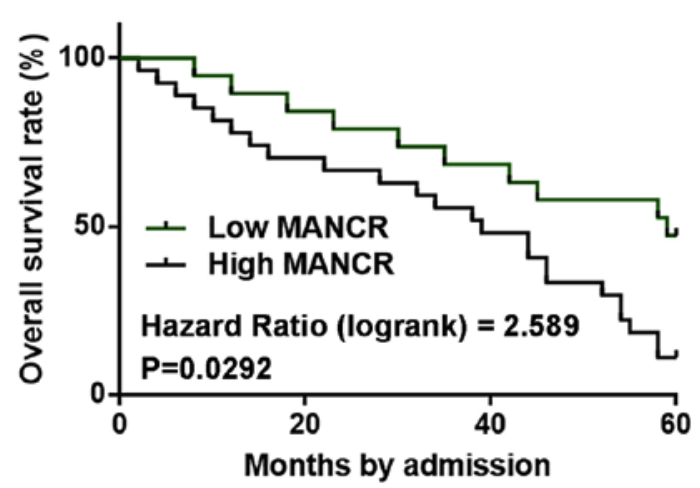

Figure 2. Patients with a high MANCR expression level exhibited a poor prognosis. Survival curve analysis revealed that patients with high MANCR expression had a significantly worse overall survival rate compared with patients with low expression. MANCR, mitotically associated long non-coding RNA.

Transcription kit (Thermo Fisher Scientific, Inc.) was used to perform reverse transcription and qPCR was subsequently performed using TaqMan Real-Time PCR Master Mix (Thermo Fisher Scientific, Inc.). miR-101 expression was normalized to the U6 endogenous control. Primer sequences were as follows: 5'-CTAATCCTGTCAGGCAGCCT-3' (forward) and 5'-ATT CTCAGGCTTTCTTTGCC-3' (reverse) for MANCR; 5'-CTA CCACATCCAAGGAAGC3' (forward) and 5'-TTTCGTCAC TACCTCCCCG-3' (reverse) for human 18S rRNA. miR-101 forward primer was: 5'-CAGTTATCACAGTGCTGATG-3'. miR-101 reverse primer and U6 primers were included in the kit. The thermocycling conditions were as follows: $95^{\circ} \mathrm{C}$ for $50 \mathrm{sec}$, followed by 40 cycles of $95^{\circ} \mathrm{C}$ for $20 \mathrm{sec}$ and $60^{\circ} \mathrm{C}$ for $45 \mathrm{sec}$. All qPCR reactions were performed in triplicate and data were analyzed using the $2^{-\Delta \Delta C q} \operatorname{method}(14)$.

Transient cell transfections. Full length MANCR cDNA was inserted into a pcDNA3.1 vector (Invitrogen; Thermo Fisher Scientific, Inc.) to establish a MANCR overexpression vector. The vector was constructed by Sangon Biotech Co., Ltd. Negative control (NC) miRNA (5'-CAGGUTUACCUAUGC CCUUUGCU-3') and an miR-101 (5'-CAGUUAUCACAGUGC UGAUGCU-3') mimic were purchased from Sigma-Aldrich, 

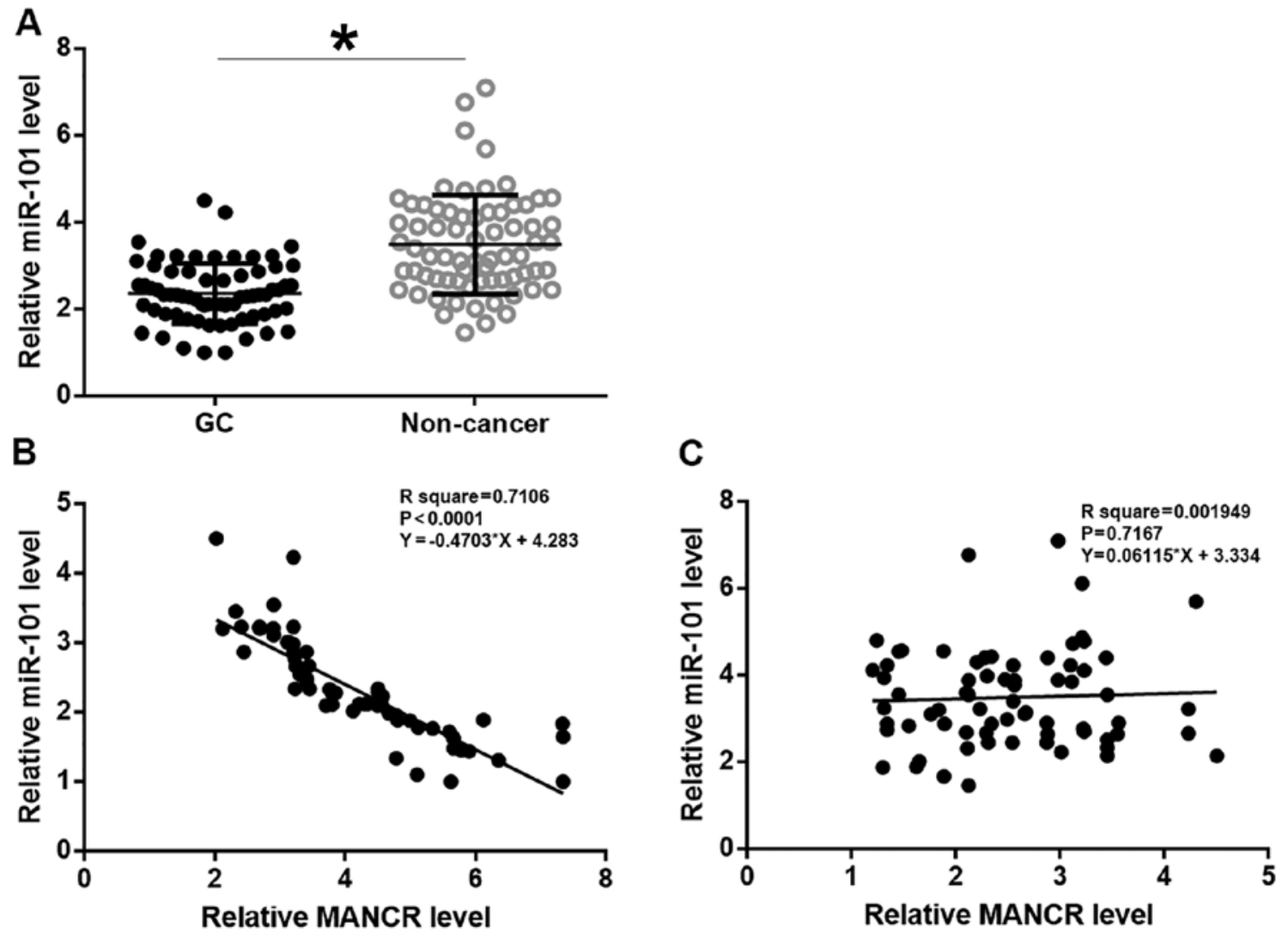

Figure 3. miR-101 expression was inversely associated with MANCR expression. (A) Analysis of reverse-transcription quantitative polymerase chain reaction data revealed that miR-101 was significantly downregulated in GC tissues compared with adjacent non-cancerous tissues in GC patients. Linear regression revealed that miR-101 was significantly and inversely associated with MANCR (B) in GC tissues but (C) not in healthy non-cancerous tissues. * $<0.05$. miR, microRNA; MANCR, mitotically associated long non-coding RNA; GC, gastric cancer.

Merck KGaA. MANCR small interfering (si) RNA (5'-GAG AACUGCCGGGACCUCCAG-3') and NC siRNA 5'-(UUG GACCCUUUCGCUGUACUC-3') were purchased from Shanghai GenePharma Co., Ltd. HGC-27 and SNU-1 cells were cultured overnight in aforementioned medium under aforementioned conditions in a 6-well cell culture medium (1x10 $0^{6}$ cells/well) overnight to reach $70-80 \%$ confluency. All cell transfections were performed using Lipofectamine ${ }^{\circledR} 2000$ reagent (Invitrogen; Thermo Fisher Scientific, Inc.), according to the manufacturer's protocols. The vector was transfected at a concentration of $10 \mathrm{nM}$ and the miRNAs and siRNAs were transfected at a concentration of $35 \mathrm{nM}$. A control (C) group consisting of untransfected cells and a $\mathrm{NC}$ group consisting of cells transfected with NC miRNA, NC siRNA or an empty vector were included. Subsequent experiments were performed $24 \mathrm{~h}$ following transfection.

Cell Counting Kit-8 (CCK-8) assay. HGC-27 and SNU-1 cells were harvested $24 \mathrm{~h}$ following transfection and single cell suspensions using EMEM were prepared. The cell density was adjusted to $4 \times 10^{4}$ cells $/ \mathrm{ml}$. Cells were subsequently cultured under aforementioned conditions in a 96 -well plate $(0.1 \mathrm{ml}$ per well). A total of $10 \mu \mathrm{l} \mathrm{CCK-8} \mathrm{solution} \mathrm{(Sigma-Aldrich;} \mathrm{Merck}$ $\mathrm{KGaA}$ ) was added to each well every $24 \mathrm{~h}$ over a four-day period. Cells were subsequently incubated under aforementioned conditions for an additional $4 \mathrm{~h}$. The optical density values were measured at a wavelength of $450 \mathrm{nM}$.
Statistical analysis. All experiments in the current study were performed in triplicate. Data were expressed as mean \pm standard deviation. Differences among different clinical stages or among different cell transfection groups were analyzed by the one-way ANOVA followed by the Tukey post hoc test. Differences between GC and adjacent non-cancerous tissues were analyzed using the paired t-test. Linear regression was used to analyze the association between the expression levels of MANCR and miR-101. Patients were divided into high and low MANCR expression level groups according to Youden's index, with 32 and 37 patients in each group, respectively. Survival curves were plotted using the Kaplan-Meier method and compared using the log-rank test. Univariate regression was performed to analyze the effects of patient clinical data on MANCR expression. $\mathrm{P}<0.05$ was considered to indicate a statistically significant difference.

\section{Results}

MANCR is upregulated in patients with GC and is not affected by clinical stages. Expression of MANCR in GC and adjacent non-cancerous tissues was analyzed by RT-qPCR. MANCR expression was significantly upregulated in GC tissues compared with adjacent non-cancerous tissues (Fig. 1A; $\mathrm{P}<0.05)$. No significant differences in MANCR expression levels were observed among patients with different clinical stages of GC (Fig. 1B). In addition, univariate regression was 

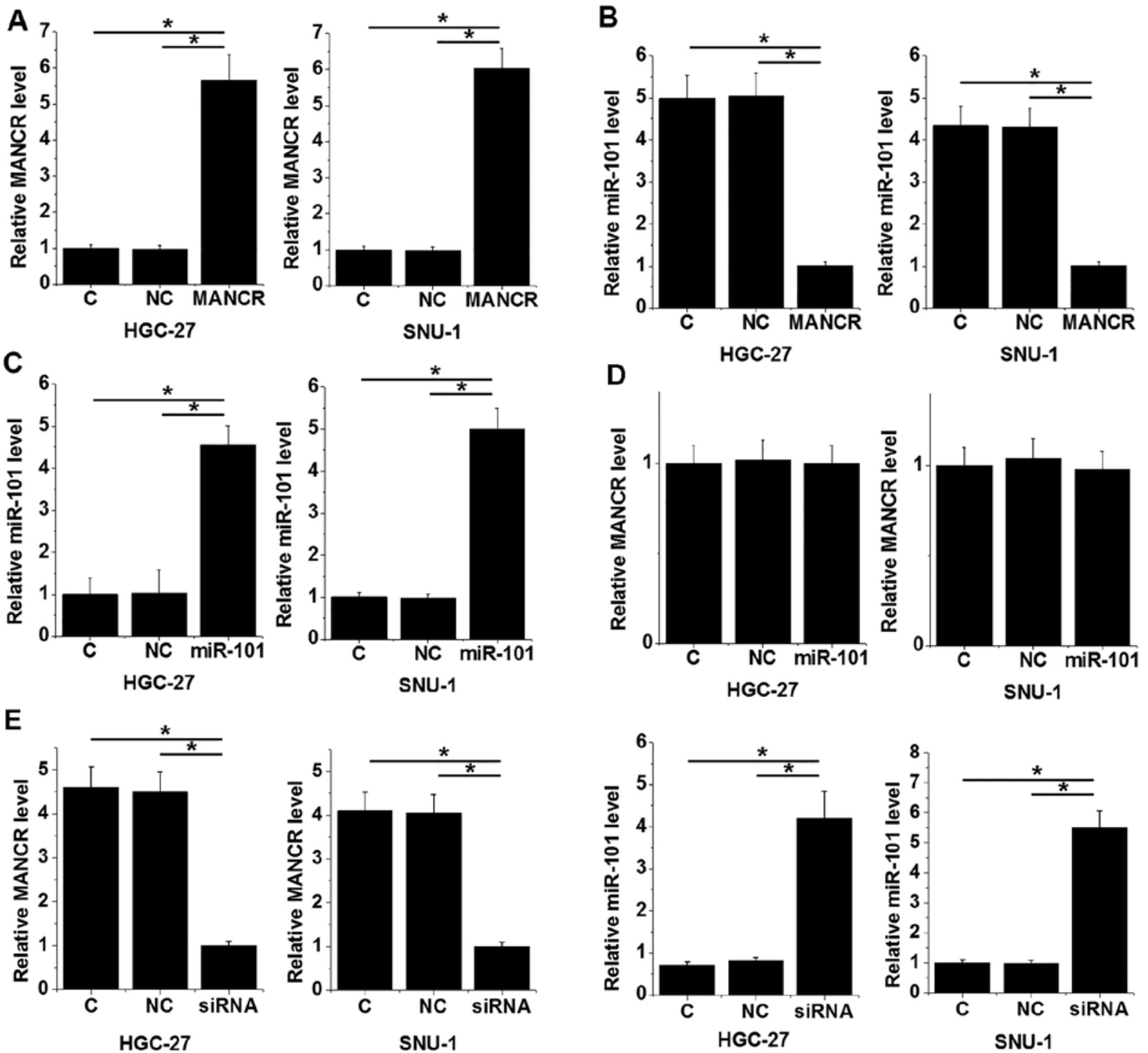

Figure 4. miR-101 expression was regulated by MANCR in GC cells. The one-way ANOVA and Tukey post hoc test revealed that expression levels of (A) MANCR and (B) miR-101 were significantly increased $24 \mathrm{~h}$ following transfection compared with the C and NC groups. (C) MANCR overexpression resulted in miR-101 downregulation in GC cells compared with the $\mathrm{C}$ and $\mathrm{NC}$ groups. (D) miR-101 overexpression did not alter MANCR expression compared with the $\mathrm{C}$ and NC groups. (E) MANCR siRNA mediated the upregulation of miR-101 compared with the $\mathrm{C}$ and NC groups. ${ }^{*} \mathrm{P}<0.05$. miR, microRNA; MANCR, mitotically associated long-non coding RNA; GC, gastric cancer; $\mathrm{C}$, control; NC, negative control; si, small interfering.
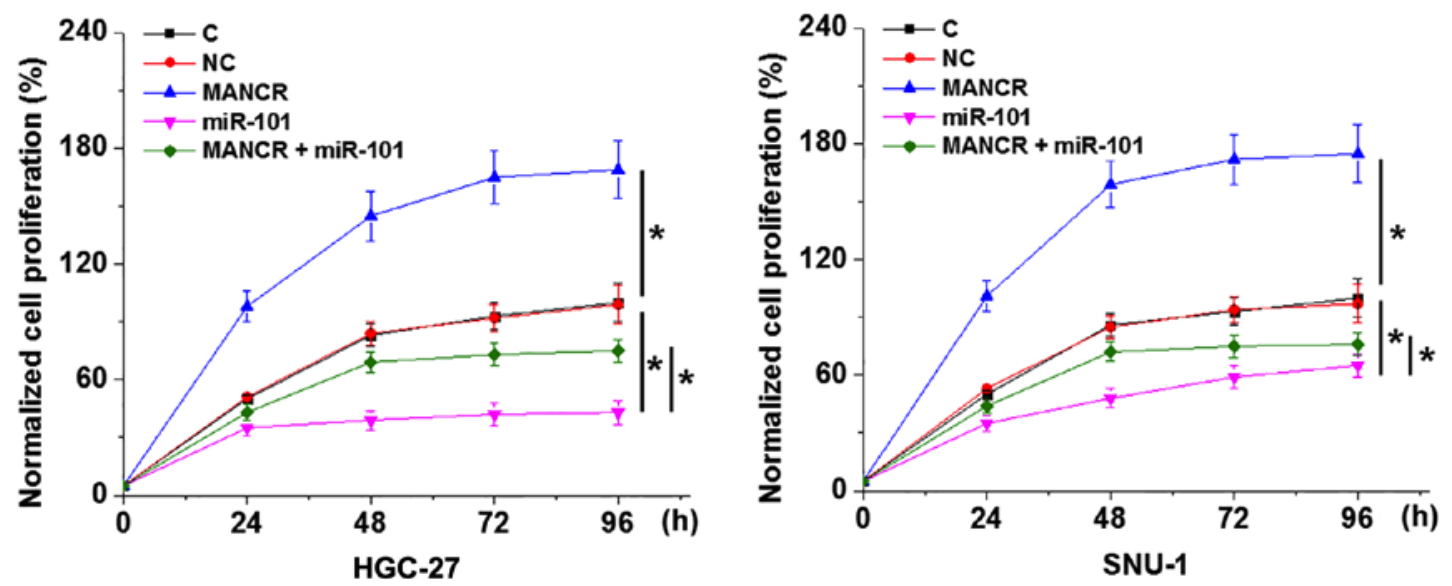

Figure 5. MANCR regulates GC cell proliferation through miR-101. Analysis of the Cell Counting Kit-8 data by the one-way ANOVA and Tukey post hoc test revealed that, compared with the $\mathrm{C}$ and NC groups, MANCR overexpression promoted, while miR-101 overexpression inhibited GC cell proliferation. Additionally, miR-101 overexpression decreased the effect of MANCR overexpression on cell proliferation. " $\mathrm{P}<0.05$. MANCR, mitotically associated long non-coding RNA; GC, gastric cancer; miR, microRNA; C, control; NC, negative control. 
performed to analyze the effects of patient clinical data on MANCR expression. Patient age, sex, smoking and drinking habits BMI and history of gastric diseases did not have significant effects on MANCR expression (data not shown; $\mathrm{P}<0.05$ ).

Patients with high MANCR expression levels exhibit worse survival compared with patients with low expression. Patients were divided into high and low MANCR expression level groups based on Youden's index, with 32 and 37 cases in each group, respectively. Survival curves were plotted using the Kaplan-Meier method and compared by the log-rank test. Patients in the high MANCR expression level group had a significantly worse overall survival rate compared with patients in the low expression group (Fig. 2; $\mathrm{P}<0.05$ ).

miR-101 is inversely associated with MANCR. Expression of miR-101 in tissues was analyzed by RT-qPCR. miR-101 was significantly downregulated in GC tissues compared with adjacent non-cancerous tissues (Fig. 3A; $\mathrm{P}<0.05$ ). Linear regression was used to analyze the association between expression levels of MANCR and miR-101. The expression level of miR-101 was significantly and inversely associated with the MANCR expression level in GC tissues (Fig. 3B; $\mathrm{P}<0.05$ ), but not in adjacent non-cancerous tissues (Fig. 3C).

miR-101 expression is regulated by MANCR in GC cells. The MANCR expression vector, MANCR siRNA and miR-101 mimic were transfected into HGC-27 and SNU-1 cell lines. The expression levels of MANCR (Fig. 4A) and miR-101 (Fig. 4B) were significantly increased $24 \mathrm{~h}$ following transfection compared with the $\mathrm{C}$ and $\mathrm{NC}$ groups $(\mathrm{P}<0.05)$, indicating successful transfection. MANCR overexpression resulted in miR-101 downregulation in the HGC-27 and SNU-1 cells compared with the $\mathrm{C}$ and $\mathrm{NC}$ groups (Fig. $4 \mathrm{C}$; $\mathrm{P}<0.05$ ), while miR-101 overexpression did not alter MANCR expression compared with the $\mathrm{C}$ and NC groups (Fig. 4D. Furthermore, MANCR siRNA resulted in miR-101 upregulation compared with the $\mathrm{C}$ and $\mathrm{NC}$ groups (Fig. 4E; $\mathrm{P}<0.05$ ).

$M A N C R$ regulates $G C$ cell proliferation through miR-101. The CCK-8 assay revealed that, compared with the $\mathrm{C}$ and NC groups, MANCR overexpression promoted, while miR-101 overexpression inhibited GC cell proliferation (Fig. 5; P<0.05). In addition, miR-101 overexpression decreased the effect of MANCR overexpression on cell proliferation (Fig. 5; $\mathrm{P}<0.05$ ).

\section{Discussion}

To the best of our knowledge, the function of MANCR has only been characterized in breast cancer (11). The present study analyzed the expression pattern of MANCR in GC and explored its effects on cancer cell behavior. MANCR may serve an oncogenic role in gastric cancer by downregulating miR-101, which has been identified as a tumor suppressive miRNA in GC (12).

Despite improvements in treatment, the overall survival rate for patients with GC remains poor $(15,16)$. Furthermore, the mortality rate in developing countries, such as China, is higher than that in Western countries, such as the United States of America (16). The present study focused on the prognostic value of MANCR in GC. MANCR was upregulated in
GC tissues compared with adjacent non-cancerous tissues but was not affected by clinical stages, suggesting that MANCR upregulation may be implicated in the development of GC. Analysis of survival data revealed that patients with high MANCR expression levels in GC tissues had a significantly decreased overall survival rate compared with patients with low MANCR expression. Therefore, MANCR may serve as a prognosis indicator in GC.

lncRNAs participate in cancer biology by regulating downstream regulatory molecules, including miRNAs $(17,18)$. The present study revealed that MANCR is a likely upstream inhibitor of miR-101 in GC cells. A previous study demonstrated that overexpression of miR-101 inhibits GC cell migration and invasion in vitro (12). The present study revealed that miR-101 overexpression inhibited the proliferation of GC cells. The results obtained in the present study are consistent with the role of MANCR in breast cancer, where it mediates cancer cell division (11). IncRNAs may serve as an miRNA sponge (19); however, a binding site for miR-101 was not found on MACNR. Further investigation is required to elucidate the interactions between MANCR and miR-101.

The present study investigated the role of MACNR in regulating cancer cell proliferation. Further studies are required to investigate the roles of this lncRNA in regulating other cancer cell behaviors, including cell cycle progression and apoptosis.

In conclusion, the results obtained in the present study revealed that MANCR was upregulated in GC tissues and promoted GC cell proliferation in vitro by downregulating miR-101.

\section{Acknowledgements}

Not applicable.

\section{Funding}

The present study was supported by the National Natural Science Foundation of China (grant no. 81660408).

\section{Availability of data and materials}

The datasets used and/or analyzed during the current study are available from the corresponding author on reasonable request.

\section{Authors' contributions}

NF and LY designed the experiments. LY, JY and LG performed experiments and data analysis. SH and FC analyzed data. All authors read and approved the final manuscript.

\section{Ethics approval and consent to participate}

Ethical approval was obtained from The Third Affiliated Hospital of Nanchang University Medical Research Ethics Committee, and written informed consent was obtained from all patients and controls.

\section{Patient consent for publication}

Not applicable. 


\section{Competing interests}

The authors declare that they have no competing interests.

\section{References}

1. Bertuccio P, Chatenoud L, Levi F, Praud D, Ferlay J, Negri E, Malvezzi M and La Vecchia C: Recent patterns in gastric cancer: A global overview. Int J Cancer 125: 666-673, 2009.

2. Jemal A, Bray F, Center MM, Ferlay J, Ward E and Forman D Global cancer statistics. CA Cancer J Clin 61: 69-90, 2011.

3. Chen W, Zheng R, Baade PD, Zhang S, Zeng H, Bray F, Jemal A $\mathrm{Yu}$ XQ and He J: Cancer statistics in China, 2015. CA Cancer J Clin 66: 115-132, 2016.

4. Zhu S, Mao J, Shao Y, Chen F, Zhu X, Xu D, Zhang X and Guo J: Reduced expression of the long non-coding RNA AI364715 in gastric cancer and its clinical significance. Tumour Biol 36: 8041-8045, 2015

5. Van Cutsem E, Sagaert X, Topal B, Haustermans K and Prenen H: Gastric cancer. Lancet 388: 2654-2664, 2016.

6. Gutschner T and Diederichs S: The hallmarks of cancer: A long non-coding RNA point of view. RNA Biol 9: 703-719, 2012.

7. Li CH and Chen Y: Targeting long non-coding RNAs in cancers: Progress and prospects. Int J Biochem Cell Biol 45: 1895-1910, 2013.

8. Bernard D, Prasanth KV, Tripathi V, Colasse S, Nakamura T, Xuan Z, Zhang MQ, Sedel F, Jourdren L, Coulpier F, et al: A long nuclear-retained non-coding RNA regulates synaptogenesis by modulating gene expression. EMBO J 29: 3082-3093, 2010.

9. Gibb EA, Brown CJ and Lam WL: The functional role of long non-coding RNA in human carcinomas. Mol Cancer 10: 38, 2011.

10. Wahlestedt C: Targeting long non-coding RNA to therapeutically upregulate gene expression. Nat Rev Drug Discov 12: 433-446, 2013.
11. Tracy KM, Tye CE, Ghule PN, Malaby HLH, Stumpff J, Stein JL, Stein GS and Lian JB: Mitotically-associated lncRNA (MANCR) affects genomic stability and cell division in aggressive breast cancer. Mol Cancer Res 16: 587-598, 2018.

12. Wang HJ, Ruan HJ, He XJ, Ma YY, Jiang XT, Xia YJ, Ye ZY and Tao HQ: MicroRNA-101 is down-regulated in gastric cancer and involved in cell migration and invasion. Eur $\mathbf{J}$ Cancer 46: 2295-2303, 2010

13. He X, Wu W, Lin Z, Ding Y, Si J and Sun LM: Validation of the American Joint Committee on Cancer (AJCC) stage system for gastric cancer patients: A population-based analysis. Gastric Cancer 21: 391-400, 2018.

14. Livak KJ and Schmittgen TD: Analysis of relative gene expression data using real-time quantitative PCR and the 2(-Delta Delta C(T)) method. Methods 25: 402-408, 2001.

15. Strong VE, Song KY, Park CH, Jacks LM, Gonen M, Shah M, Coit DG and Brennan MF: Comparison of gastric cancer survival following R0 resection in the United States and Korea using an internationally validated nomogram. Ann Surg 251: 640-646, 2010.

16. Strong VE, Wu AW, Selby LV, Gonen M, Hsu M, Song KY, Park CH, Coit DG, Ji JF and Brennan MF: Differences in gastric cancer survival between the U.S. and China. J Surg Oncol 112: 31-37, 2015

17. Jalali S, Bhartiya D, Lalwani MK, Sivasubbu S and Scaria V: Systematic transcriptome wide analysis of lncRNA-miRNA interactions. PLoS One 8: e53823, 2013

18. Liz $\mathrm{J}$ and Esteller M: lncRNAs and microRNAs with a role in cancer development. Biochim Biophys Acta 1859: 169-176, 2016.

19. Liang WC, Fu WM, Wong CW, Wang Y, Wang WM, Hu GX, Zhang L, Xiao LJ, Wan DC, Zhang JF and Waye MM: The lncRNA H19 promotes epithelial to mesenchymal transition by functioning as miRNA sponges in colorectal cancer. Oncotarget 6: 22513-22525, 2015. 